\title{
sur la gestion et les finances publiques En Août 2020
}

\section{Budget de L'ÉTAT}

\section{Données générales sur les finances publiques}

La moitié du coût de la crise au $2^{\mathrm{e}}$ trimestre supportée par les APU

Dans sa note du 28 août sur les comptes nationaux, I'INSEE évalue la perte de PIB au $2^{\mathrm{e}}$ trimestre 2020, par rapport à la même période de 2019, à $90 \mathrm{Md}$ dont 50,9 Md€ (56\%) supportés par les administrations publiques en raison des moindres recettes fiscales et de la hausse des prestations financées par le déficit $\left(11,6 \%\right.$ du PIB au $2^{2}$ trimestre). La perte des entreprises est évaluée à $36 \mathrm{Md} €$ hors reports de charges et prêts garantis qui sont considérés comme dues ou remboursables. Le revenu des ménages n'a baissé que de 2,3\% (4 Md€). Comme les dépenses de consommation ont chuté de $-11,5 \%$, le taux d'épargne a fait un bond $27,4 \%$.

\section{Dette publique}

Morgan Stanley suspendue du club des SVT

L'Agence France Trésor (AFT) a annoncé le 3 août avoir décidé de suspendre la banque américaine Morgan Stanley de son statut de spécialiste en valeurs du Trésor (SVT) pour au moins trois mois à compter du 4 août 2020 en raison de transactions effectuées le 16 juin 2015 ayant eu pour effet de porter gravement atteinte à la liquidité du marché des obligations souveraines françaises. La banque a fait appel de la sanction. Les 14 autres SVT s'activent avec l'AFT pour mener à bien le programme de 260 Md€ d'émissions en 2020.

\section{FinANCES LOCALES}

\section{$\pi$ Dépenses publiques locales}

La budgétisation et la comptabilisation des dépenses liées au COVID

Le 24 août 2020, a été publiée la circulaire relative au traitement budgétaire et comptable des dépenses des collectivités liées à la gestion de la crise sanitaire du Covid-19. Celle-ci prévoit un certain nombre d'adaptations. Parmi les éléments importants, les collectivités pourront étaler sur cinq ans certaines dépenses relatives à la gestion de la crise sanitaire, intervenues entre le début de l'état d'urgence sanitaire (soit le 24 mars) et la fin de l'exercice 2020. De façon un peu surprenante, ces dépenses en grande partie de fonctionnement pourront être financées par l'emprunt. Les collectivités pourront, sur la base d'une délibération motivée en raison des conséquences de la crise sanitaire, et après avis du comptable, procéder à la reprise $d^{\prime}$ un excédent $d^{\prime}$ 'investissement en section de fonctionnement. Enfin, notons une annexe budgétaire au compte administratif devra être élaborée devant permettre aux collectivités qui le souhaitent d'identifier les dépenses de fonctionnement et d'investissement relatives à la crise sanitaire.

\section{त Comptabilité publique locale}

Suppression de l'indemnité de conseil des comptables publics

Deux arrêtés adoptés 20 août 2020 (un remplaçant celui du 16 décembre 1983 et un autre remplaçant celui du 12 juillet 1990) traduisent règlementairement la fin d'une époque, déjà actée en loi de finances pour 2020. A la suite du vote de cette loi, les collectivités territoriales 
ne versent plus d'indemnités dites de conseil à leurs comptables assignataires, mode de fonctionnement qui pouvait à certains égards apparaître désuet. Cependant, il ne faudrait pas croire que le montant ainsi dévolu aux comptables a disparu. Il a été compensé par l'État à ces derniers, celui-ci se refinançant indirectement sur le dos des collectivités avec une baisse des variables d'ajustement de l'ordre de 25 millions d'euros.

\section{FINANCES SOCIALES}

\section{$\lambda$ Politique sociale}

Plan de relance, volet social : premières annonces

L'annonce du contenu du plan de relance de $100 \mathrm{Md} €$ a été retardée au conseil des ministres du 2 septembre. Mais devant I'Université d'été du MEDEF, le 26 août, le Premier Ministre a dévoilé un certain nombre d'éléments, notamment les allègements fiscaux en faveur des entreprises (baisse de 10Md€ des impôts de production, poursuite de l'allègement de l'impôt sur les sociétés...). II a également indiqué que la diminution de 85 à $60 \%$ de la prise en charge par l'État du coût du chômage partiel pour les entreprises, initialement fixée au $1^{\text {er }}$ octobre 2020, serait repoussée au 1er novembre. Le bénéfice de la prise en charge du taux à $85 \%$ sera maintenu jusqu'au 1er janvier 2021 pour les secteurs de l'événementiel, du tourisme et de la restauration et porté jusqu'à 2 ans pour les entreprises ayant conclu un « accord d'activité partielle de longue durée ».

\section{$\lambda$ Dette sociale}

Promulgation de la loi du 7 août 2020 relative à la dette sociale et à l'autonomie

La loi organique $n^{\circ} 2020-991$ du 7 aoùt 2020 relative à la dette sociale et à l'autonomie a été publiée au JO du 8 août. Conformément à ce qui avait été annoncé (v. Repères de mai), elle prévoit la prolongation de la CADES jusqu'en 2033 pour financer le surcroît de dette sociale lié à la crise du COVID-19, ainsi que le principe d'une présentation annuelle des financements de la Sécurité sociale destinés au soutien à l'autonomie des personnes âgées et des personnes en situation de handicap.

\section{$\lambda$ Dépenses de santé/hôpital/assurance maladie}

Le gouvernement envisage de taxer les complémentaires santé
Les assureurs santé auraient réalisé un montant de près de $3 \mathrm{Md}$ d€€ d'économies de remboursements du fait d'un moindre recours aux soins médicaux pendant la période du confinement. Le gouvernement envisage de mettre en place une «contribution exceptionnelle» pour permettre de financer une partie du déficit massif que va enregistre l'Assurance maladie (-31 Md€) en 2020. Les complémentaires santé font valoir qu'elles subissent l'effet des moindres rentrées de cotisations du fait de la crise économique et d'un rattrapage des dépenses de santé à la sortie du confinement, ce qui pourrait amener à procéder par étapes pour la mise en œuvre de ce prélèvement.

Une prime Covid pour les professionnels à domicile

Le gouvernement s'est engagé le 4 août sur une contribution de $80 \mathrm{ME}$ pour verser une prime exceptionnelle non imposable aux professionnels de l'aide et de l'accompagnement à domicile, qui, à la différence des autres professionnels de santé, n'avaient pas bénéficié jusqu'à présent de mesure de reconnaissance pour leur engagement dans la crise du COVID-19. Avec la contribution des départements, cet engagement de l'État doit permettre le versement d'une prime de $1000 €$ prorata temporis à verser d'ici la fin 2020.

\section{$\pi$ Politique d'allègement des charges et du cout du travail}

\section{Décret sur la prime à l'embauche pour} l'emploi des jeunes

Le décret $n^{\circ} 2020-982$ du 5 août 2020 instituant une prime à l'embauche pour l'emploi des jeunes de moins de 26 ans a été publié au JO du 6 août. Mesure phare du plan « 1 jeune, 1 solution » annoncé le mois dernier (cf Repères de juillet), le texte prévoit une prime d'un montant maximal de $4000 €$ pour toute embauche d'ici le 31 janvier 2021 d'un salarié de moins de 26 ans dont le salaire mensuel n'excéderait pas 2 SMIC (3 $080 €$ bruts). Le gouvernement table sur un recours à 450000 primes, pour un montant de l'ordre de 1,5 Md€, soit le quart environ du montant total prévu pour le plan d'emploi des jeunes. Le bénéfice de cette prime serait accordé sans limite d'âge pour l'embauche de travailleurs en situation de handicap et il serait cumulable avec tout autre dispositif de soutien pour l'emploi de ces personnes (aides de l'Agefiph). 


\section{त Solidarité/lutte contre la pauvreté/ revenu universel}

L'aide exceptionnelle de solidarité et le coût du confinement

Le $1^{\text {er }}$ août, un blog de l'OFCE fait le point sur I'efficacité de l'aide exceptionnelle mise en place en avril dernier (cf REPĖRES d'avril) au profit des ménages éligibles au RSA, à l'ASS et aux ménages avec enfants bénéficiaires d'aides au logement. Ces aides, qui ont pu être complétées par les collectivités locales, devaient compenser les coûts supplémentaires entrainés par le confinement (alimentation des enfants privés de cantines scolaires, gardes à domicile pour les parents ne pouvant pas télétravailler, dépenses d'informatique...), ainsi que la potentielle perte de revenu liée au basculement dans le chômage partiel. II résulte de cette analyse que l'aide nationale et locale a permis de compenser l'augmentation du prix des repas pour les familles les plus modestes (sujet le plus sensible). En revanche, elles n'ont pas compensé la perte de revenus pour les ménages subissant un chômage partiel lorsque leur rémunération était supérieure au SMIC horaire, même si cet effet a pu être atténué par le jeu de la prime d'activité. En revanche pour les personnes qui ont perdu leur emploi, qui bénéficient de l'Allocation de retour à l'emploi, ce mécanisme n'a pas pu jouer, ce qui a pu faire basculer un certain nombre de chômeurs au-dessous du seuil de pauvreté.

\section{त Autonomie/dépendance}

Un rapport de l'IGAS sur l'emploi des handicapés

L'IGAS a remis au Président de la République le 17 août un rapport sur « Handicaps et emploi ». Ce rapport constate l'essoufflement des dispositifs en faveur de l'emploi des personnes en situation de handicap qui repose essentiellement sur l'obligation d'emploi d'au moins $6 \%$ de travailleurs handicapés, puisque ce taux n'est pas atteint plus de 30 ans après l'instauration du dispositif et que le taux de chômage de ces travailleurs reste le double de celui de la population générale (18\%), avec même un accroissement de la discrimination, comme le constate le Défenseur des droits. II critique une approche trop individuelle et médicale de la situation du travailleur handicapé, alors que les approches internationales d'aujourd'hui mettent l'accent sur l'inclusion. Le rapport propose enfin trois orientations : le statu quo avec des aménagements, un recentrage sur des dispositifs plus ci- blés, et une refondation en profondeur, qui évaluerait le handicap non en fonction de critères personnels, mais à partir de l'environnement de travail aménagé selon le principe de l'accessibilité universelle. Le gouvernement a par ailleurs étendu le bénéfice de la prime à l'embauche pour ces personnes (cf supra).

\section{FisCALITÉ ET PROCÉDURE FISCALE}

\section{$\lambda$ Impôt sur les sociétés}

"Accord Facebook » sur un redressement d'IS de $106 \mathrm{M} €$

Une convention entre la DGFIP et la filiale Facebook France sur un redressement de $106 \mathrm{M€}$ d'IS sur la période 2009-2018 a été signée. Si I'administration fiscale est demeurée discrète sur cet accord, ladite société a précisé : « nous prenons nos obligations fiscales au sérieux, nous payons les impôts que nous devons dans tous les marchés où nous opérons et travaillons étroitement avec les administrations fiscales à travers le monde pour assurer le respect de toutes les lois fiscales applicables et résoudre tout litige ». Au titre de l'exercice 2019, la filiale Facebook France a annoncé s'être acquitté de 8,46 M€ d'IS, soit une hausse de $50 \%$ par rapport à l'exercice 2018, ce qui demeure très modeste par rapport aux 6,3 Md€ d'impôts sur les bénéfices annoncés par le groupe Facebook dans ses comptes annuels pour l'exercice 2019, acquittés principalement aux USA. Il est évident que le marché économique français contribue nécessairement davantage aux bénéfices de celle-ci. Depuis 2017, le chiffre d'affaires généré par les équipes de vente localisées en France est désormais déclaré et frappé en France en étant intégré dans le calcul de l'IS de Facebook France. Rappelons aussi que des négociations sont en cours au sein de I'OCDE pour un accord sur la taxation des GAFA (v. Repères de mai 2020). Depuis 2017, plusieurs contentieux fiscaux avec les GAFA ont été soldés avec la DGFIP (Amazon, févr. 2018, 200 M€; Apple, janv. $2019500 \mathrm{M€}$; Google, sept. 2019, 1 Mds€).

\section{Politique fiscale}

Fondation Jean Jaurès : un manifeste pour repenser la fiscalité française?

Un rapport de la Fondation Jean Jaurès, réservoir d'idées proche du Parti socialiste, propose quelques pistes pour une imposition plus simple et plus équitable. Le constat est partagé 
autour du consensus quant à la complexité du système fiscal français, source d'injustices. Ce manifeste propose des mesures visant à une hausse de la fiscalité des ménages les plus aisés, à contre-courant de la politique fiscale menée par le Gouvernement depuis 2017 (baisse de I'IR, réforme de I'IFI, suppression de la TH, création de la "flat tax», etc.). Première piste : renforcer la progressivité de I'IR en créant de nouvelles tranches afin d'imposer plus fortement les ménages à hauts revenus. Deuxième piste: une fusion de I'IR et de la CSG en rendant cette dernière plus progressive. Troisième piste : supprimer le foyer fiscal afin d'assurer l'individualisation de I'IR, mesure rendue techniquement plus simple grâce à la mise en place du prélèvement à la source. Quatrième piste : supprimer les niches fiscales (on en dénombre en France pas moins de 470 pour un coût estimé à 100 Mds€ chaque année) sauf dans l'hypothèse où leur pertinence aurait été explicitement démontrée et leur prorogation votée par le Parlement.

PLF 2021, les mesures d'allègement fiscal pour les entreprises

Lors de l'université d'été du MEDEF, le Premier ministre a annoncé plusieurs mesures fiscales en faveur des entreprises qui devraient figurer au PLF 2021. Le Gouvernement a décidé de baisser les impôts de production de $10 \mathrm{Mds} €$ par an (v. Repères de juillet) autour de trois volets : une division par deux de la CVAE avec suppression de la part régionale; une baisse du plafonnement de la CET en part de la valeur ajoutée de $3 \%$ à $2 \%$; une réforme de la méthode de taxation foncière des locaux industriels afin de réduire la pression fiscale sur ces locaux. Le Gouvernement continuera à baisser I'IS ainsi que s'y est engagé le Président de la République afin d'assurer la stabilité des règles du jeu fiscal, condition essentielle de la confiance économique.

\section{$\lambda$ Procédure fiscale}

Mise à jour de la Charte des droits et obligations du contribuable vérifié

La DGFIP a mis à jour la charte des droits et obligations du contribuable vérifié qui énonce les garanties dont il bénéficie lorsqu'il fait l'objet d'une vérification de comptabilité, d'un examen de comptabilité ou d'un examen contradictoire de situation fiscale personnelle. Les dispositions contenues dans cette charte, mise à jour annuellement, sont opposables à la DGFIP (art. L. 10 et L. 47 du LPF).

\section{Management public}

\section{Relation avec les usagers}

Mise en service du paiement des factures publiques chez les buralistes

Un communiqué et un dossier de presse présentent le nouveau dispositif de paiement de proximité, autorisé par l'article 201 de la loi de finances pour 2019 et confié au réseau des buralistes pour une durée de cinq ans. Toutes les factures publiques de la vie quotidienne (cantine, crèche, hôpital, amendes forfaitaires et majorées... sans limite de montant) et les impôts jusqu'à $300 €$ seront concernées par ce dispositif qui permettra d'éliminer les paiements en espèces aux guichets de la DGFiP. 5100 points de paiement sont agréés, répartis dans 3400 communes dont 1600 où la DGFiP n'est pas (ou plus) présente aujourd'hui.

\section{$\lambda$ Administration numérique}

L'observatoire de la qualité de la dématérialisation

La direction interministérielle du numérique a mis en ligne la dernière édition de l'observatoire de la qualité des services numériques. Sur 250 démarches courantes, 180 sont effectivement dématérialisées. En progrès : le bouton "je donne mon avis » a été utilisé par les usagers pour 102 procédures; en retard : 22 procédures seulement sont effectivement accessibles aux handicapés.

\section{Stop Covid à faible régime}

L'application Stop Covid (v. Repères d'avril, mai et juin 2020) a été téléchargée par 2,3 millions de personnes, soit 3,4 \% de la population. 1725 personnes se sont signalées positives. 103 personnes ont été prévenues d'un éventuel contact positif grâce à cette application. Le coût de l'opération est évalué entre $120000 €$ et $200000 €$ par mois. D'autres pays d'Europe ont lancé des dispositifs de traçage « décentralisés » mais fournis partiellement par Google et Apple. Le taux de téléchargement atteint $38 \%$ en Islande, $30 \%$ en Irlande, $25 \%$ en Suisse, près de $20 \%$ en Allemagne. On estime que ce type d'application n'est vraiment efficace que si elle est utilisée par $60 \%$ de la population. En France, alors que la CNIL a demandé au gouvernement d'évaluer et d'établir l'utilité du dispositif, on annonce une version plus ergonomique de l'application. 


\section{त Administration territoriale}

Les DDI rattachées au ministère de l'intérieur

Les directions départementales interministérielles (territoires, cohésion sociale, protection des populations), déjà placées sous l'autorité des préfets, sont rattachées au ministère de l'intérieur par un décret $n^{\circ}$ 2020-1050 du 14 août 2020, publié au JORF du 15 août, texte $n^{\circ} 30$. Ce ministère conduira et animera le réseau des $\mathrm{DDI}$ « en liaison avec les ministères concernés et dans le respect de leurs attributions respectives ». Environ 1000 fonctionnaires des services déconcentrés changent ainsi de ministère.

Des sous-préfets à la relance et simplifications

La nouvelle ministre de la transformation et de la fonction publiques a annoncé le 30 août la désignation de sous-préfets à la transformation et à la relance chargés de contribuer à la mise en œuvre du plan de relance et de lever les «blocages administratifs». Ils ne seront pas désignés dans tous les territoires et leur rattachement aux préfets de départements ou de région n'est pas encore tranché. Ce dispositif rappelle les commissaires au redressement productif mis en place au lendemain de la crise financière de 2009. Le Premier ministre a indiqué que le Plan de relance sera accompagné d'un effort particulier de simplification : guichet unique au ministère de l'économie, des finances et de la relance; allègement de certaines obligations des entreprises; relèvement des seuils de marchés publics; pérennisation de certaines mesures d'urgence.

\section{Gestion publique}

Le point sur la politique immobilière de l'État

Le rapport d'activité 2019 de la direction de l'immobilier de l'État présente les différentes activités de cette direction créée en 2016 au sein de la DGFiP pour mettre en œuvre la politique immobilière de l'État incluant la gestion du domaine. Le patrimoine se compose de 191000 bâtiments et 30500 terrains représentant $66 \mathrm{Md} €$ d'actifs. Parmi les orientations récentes: un programme de rénovation de 39 cités administratives, une contribution à la transition énergétique, 704 cessions immobilières d'un montant de $625 \mathrm{M} €$ en 2019, la réflexion sur une diversification des modalités de valorisation du patrimoine par la recherche d'alternatives à la cession telle que les baux emphytéotiques. Depuis la loi du 8 janvier 2013, la cession de 115 terrains domaniaux avec décote a permis la construction de 11000 logements dont 8000 logements sociaux.

\section{$\pi$ Fonction publique}

\section{Les finances publiques aux concours de I'ENA}

Les sujets suivants (Réponse à trois questions appuyées par quelques documents) ont été posés aux concours d'entrée à l'Ecole nationale d'administration qui se sont déroulés du 22 au 28 août : "Concours externe : La cessation d'actifs publics: enjeux et perspectives ; La suppression de la taxe d'habitation: modalités et impact sur les finances publiques locales; La gratuité des services publics de secours. Concours interne: A quoi servent les lois de finances rectificatives? Peut-on verdir le budget de la France? ; Les départements peuvent-ils faire face à leurs dépenses ? Troisième concours : Le poids des prélèvements obligatoires est-il excessif? A quoi servent les dépenses fiscales? ; Faut-il donner de l'ampleur au budget de l'Union européenne? ». De quoi inciter les candidats et leurs enseignants à s'abonner à Gestion \& Finances Publiques!

\section{EUROPE}

\section{$\lambda$ Budget européen}

Le Parlement européen s'oppose au CFP amoindri par le Sommet européen

Dans une résolution adoptée à une très large majorité le 23 juillet, le Parlement européen a dénoncé les coupes « inacceptables » opérées par les États membres dans le projet de cadre financier pluriannuel (CFP) proposé par la Commission et qui ont permis de le compresser de 1300 à moins de 1100 milliards d'euros pour les sept années 2021-2028. Les eurodéputés ont ainsi menacé de ne pas approuver l'accord trouvé lors du sommet si le budget n'était pas " amélioré », notamment en exigeant - par le biais d'une lettre des présidents des principaux groupes adressée le 26 août à la chancelière allemande et à la présidente de la Commission - que le respect de l'état de droit soit intégré dans les négociations.

\section{$\pi$ Euro}

Nouveaux rebondissements dans le feuilleton BCE - Karlsruhe

L'ultimatum de la Cour constitutionnelle allemande à la Banque centrale européenne a expiré ce mercredi 5 août. Lundi, la Bundesbank 
s'était déclarée satisfaite des informations apportées par la BCE. Mais il n'est pas certain que les plaignants rendent les armes. "Naturellement, la question du PEPP va aussi se retrouver devant la Cour de Karlsruhe, et si I'on applique les critères - très restrictifs - du jugement, les choses seront serrées ", redoute Lucas Guttenberg, chef adjoint de la recherche au bureau berlinois de l'Institut Jacques Delors.

\section{Pas de succès pour les prêts du MES}

Quoique le débat soit tendu en Italie, les pays de la zone euro les plus touchés par la crise (Portugal, Espagne, Grèce et Italie) n'ont pas sollicité le Mécanisme européen de stabilité depuis le 15 mai 2020. Ainsi la presse constate début août que les 240 milliards d'euros à distribuer en lignes de crédit n'ont pas été utilisés, malgré les conditions plus qu'avantageuses proposées.

Mario Draghi recommande une "bonne dette »

Mardi 18 août, à l'occasion d'une conférence, I'ancien président de la Banque centrale européenne a déclaré que c'est seulement si la dette «sans précédent», créée par la pandémie du nouveau coronavirus, était « remboursée», qu'elle sauverait l'économie européenne.

\section{7 États membres}

L'Allemagne va dépasser à nouveau le ratio constitutionnel d'endettement

Vendredi 20 août 2020, le ministre des finances Olof Scholz a annoncé que le gouvernement va mettre de côté la politique du "Schwarz Null » (zéro déficit) en prévision d'un déficit budgétaire en 2021 pour surmonter la crise. II prévoit aussi de demander une exception à la règle sur la limitation de l'endettement public, règle appelée "frein à l'endettement», introduite dans sa Constitution en 2011 et qui interdit en principe au gouvernement fédéral d'emprunter chaque année plus de 0,35\% de son produit intérieur brut.

\section{INTERNATIONAL}

\section{ר États}

\section{Accord entre l'Argentine et ses créanciers}

Le 4 août à 3 heures du matin, après des mois de négociations, le président Fernández a annoncé que le gouvernement argentin est parvenu à un arrangement sur la restructuration de 66 milliards de dollars de sa dette publique émis sous législation étrangère. Après une première offre de restructuration présentée fin avril sur la base d'un moratoire sur les paiements jusqu'en 2023, la cinquième et dernière prévoyant finalement que les premiers remboursements d'intérêts aient lieu dès 2021 a été acceptée. La valeur nominale des titres a par ailleurs été nettement améliorée par le gouvernement, de plus de 54 dollars par tranche de 100 , contre 39 au début des négociations.

\section{Fed : I'objectif d'inflation assoupli}

Le 27 août 2020, dans un communiqué diffusé juste au début de l'intervention de son président Jérôme Powell à l'université d'été des banquiers centraux à Jackson Hole, la banque centrale américaine (Fed) a annoncé deux changements majeurs dans les règles qui définissent son action. Le FOMC, I'organe de la Fed qui pilote la politique monétaire, inverse les priorités depuis la période Volcker inaugurée en 1980 : le chômage devient la première préoccupation. Symétriquement, au lieu de cibler une inflation à $2 \%$ I'an, elle cherchera une inflation modérément supérieure à $2 \%$ pendant un certain temps. Toute la presse a ainsi constaté une révolution, petite ou pas.

Aurélien BAUDU (fiscalité et procédure fiscale), Fabrice BIN (Europe, international), Jean-François CALMETTE (budget de l'État et des opérateurs),

Matthieu HOUSER (finances locales), Michel LE CLAINCHE (comptabilité publique, management public, coordination), Yves TERRASSE (finances sociales).

\footnotetext{
Retrouvez sur www.gestionfinancespubliques. info :

-l'intégralité des REPÈRES d'actualité des finances et de la gestion publiques depuis 2015 -le 15 du mois, les REPÈRES d'actualité du mois précédent

-le 15 janvier 2020, le BEST OF des repères d'actualité de l'année 2019.

- et, en continu, les réflexions des blogueurs de G\&FP.
} 